\title{
Variaciones fenotípicas de las características textiles de fibra predescerdada de vicuña
}

\author{
Phenotypic variations of the textile characteristics of pre-dehaired fibre of vicuña
}

\author{
Rubén Pinares $^{1,3}$, Wilder Vladimir Yauri ${ }^{2}$
}

\section{Resumen}

El objetivo del estudio fue evaluar el efecto de la edad y el sexo de la vicuña sobre el diámetro medio de fibra (DMF), finura al hilado, índice de curvatura, longitud de fibra y perfil del diámetro de fibra; asimismo calcular las correlaciones fenotípicas entre las características textiles de fibra. Muestras predescerdadas $(n=88)$ fueron analizadas con el equipo OFDA 2000. En el modelo estadístico se consideró como efectos fijos a la edad, el sexo y la interacción edad:sexo. El sexo tuvo efecto significativo sobre las variaciones fenotípicas del DMF, perfil de diámetro máximo y mínimo; asimismo, la edad tuvo efecto significativo sobre el DMF, finura al hilado y perfil de diámetro mínimo. Sin embargo, la interacción de sexo y edad no tuvo efecto sobre las características estudiadas. Las correlaciones fenotípicas fueron significativas y positivas entre DMF y finura al hilado, finura al hilado y desviación estándar de diámetro, desviación estándar y coeficiente de variación de diámetro, desviación estándar de diámetro y diferencia de diámetro en perfil de fibra. Las correlaciones fenotípicas fueron negativas: porcentaje de fibras $<30 \mu \mathrm{m}$ con desviación estándar de diámetro, coeficiente de variación de diámetro y finura al hilado. Además, existe variabilidad fenotípica de DMF y perfil del diámetro de fibra en las vicuñas adultas y juveniles. La edad, el sexo y el predescerdado influyeron sobre algunas características relacionadas a la finura y el confort de la fibra.

Palabras clave: vicuña; fibra fina; longitud de fibra; perfil de diámetro de fibra

\section{Abstract}

The aim of this study was to evaluate the effect of the age and sex of the vicuña on the mean fibre diameter (MFD), spinning fineness, curvature index, fibre length and fibre diameter profile; also, to calculate the phenotypic correlations between fibre textile characteristics. Pre-dehaired samples $(n=88)$ were analysed with the OFDA 2000

\footnotetext{
${ }^{1}$ Universidad Nacional Agraria La Molina, Lima, Perú

${ }^{2}$ KAMY Textiles S.A.C., Lima, Perú

${ }^{3}$ E-mail: zoovet_p@hotmail.com
}

Recibido: 19 de marzo de 2019

Aceptado para publicación: 22 de noviembre de 2019 
equipment. In the statistical model the age, sex and age:sex interaction were considered as fixed effects. Sex had a significant effect on the phenotypic variations of MFD, maximum and minimum diameter profile; likewise, age had a significant effect on MFD, spinning fineness and minimum profile diameter. However, the interaction of sex and age had no effect on the characteristics studied. The phenotypic correlations were significant and positive between DMF and spinning fineness, spinning fineness and standard deviation of diameter, standard deviation and coefficient of variation of diameter, standard deviation of diameter and diameter difference in fibre profile. Phenotypic correlations were negative: percentage of fibres $<30 \mu \mathrm{m}$ with standard deviation of fibre diameter, coefficient of variation of diameter, and spinning fineness. In addition, there is phenotypic variability of MFD and fibre diameter profile in adult and juvenile vicuñas. Age, sex and pre-dehairing affected some fibre characteristics related to the fineness and comfort of the fibre.

Key words: vicuña; fine fibre; fibre length; fibre diameter profile

\section{INTRODUCCIÓN}

La vicuña peruana (Vicugna vicugna mensalis) se caracteriza por tener un patrón de pigmentación más intensa en el vellón y es de menor tamaño que la subespecie Vicugna vicugna vicugna (Quispe et al., 2009; Wheeler y Laker, 2009; Quispe et al., 2014). Desde tiempos ancestrales las vicuñas se capturaban en los chakus, donde las esquilaban y transformaban artesanalmente la fibra. En la actualidad, la vicuña es conocida como la «reina de los Andes» por Loro Piana (Piazza, 2013), que por ser una fibra muy fina ( «fibra de oro) compite con otras fibras como la cachemira (McGregor, 2018), mohair y seda. Además, la fibra de vicuña es muy apreciada por su excelente calidad y color natural (canela), que se cotiza entre $350 \mathrm{y}$ 1400 dólares $/ \mathrm{kg}$ de fibra sucia o fibra lavada y descerdada en la industria textil internacional (Quispe et al., 2014; Kasterine y Lichtenstein, 2018). El Perú exporta más de $5000 \mathrm{~kg} /$ año de fibra sucia a Italia (Kasterine y Lichtenstein, 2018).

La vicuña es el recurso genético más importante del Perú y es Patrimonio Nacional por su importancia económica y sociocultural. El aprovechamiento sostenible y comercialización de la fibra de vicuña ofrece una gran oportunidad de desarrollo económico para las comunidades campesinas que tienen en usufructo a esta especie. El vellón de la vicuña es doble capa, compuesto por fibras gruesas (fibras objetables) y fibras finas o down, que se originan de los folículos secundarios (Quispe et al., 2014; Chamut et al., 2016). Las principales características que determinan el valor comercial y el rendimiento textil son el diámetro medio de fibra (DMF) y su variabilidad (CV); así mismo, la longitud es una característica importante para someter al cardado o peinado (Trejo et al., 2009).

Las características que determinan la calidad textil de la fibra de vicuña han sido estudiadas en algunas regiones del Perú (Quispe, 2010; Olaguivel-Flores et al., 2018; Quispe et al., 2018). No obstante, aún no se han investigado las características textiles de la fibra de vicuña en la región de Junín. En consecuencia, el objetivo de este trabajo fue determinar el efecto de la edad y sexo sobre las principales características textiles de la fibra de vicuña: DMF, perfil de diámetro, finura al hilado, longitud e índice de curvatura de fibra. Así mismo, calcular las correlaciones fenotípicas entre las principales características textiles de fibras predescerdadas. 


\section{Materiales y Métodos}

\section{Sistema de Crianza y Chaku}

El trabajo de investigación se realizó en Cormura y Pampa Victoria, parte de la Comunidad Campesina Villa de Junín, distrito, provincia y región de Junín, Perú, ubicado a una altitud de $4105 \mathrm{msnm}$. Las vicuñas fueron manejadas bajo un sistema de semicautiverio, pastoreando en praderas naturales, dentro de un cerco perimétrico de alambrado, con una extensión total de 1800 ha. La zona está monitoreada y vigilada por los guardaparques, con la supervisión del Servicio Nacional Forestal y de Fauna Silvestre (SERFOR) y la Agencia Agraria de Junín, adscrito al Ministerio de Agricultura y Riego (MINAGRI).

El «chaku» de vicuñas del presente estudio se realizó en setiembre de 2013, con el fin de capturar, esquilar y tomar muestras de fibra de hembras vacías cuya longitud de mecha era mayor a $2 \mathrm{~cm}$, sin caspa. Las hembras preñadas no se esquilaron debido a procedimientos establecidos de bienestar animal.

\section{Animales y Análisis de Muestras}

La Comunidad Campesina Villa de Junín tiene más de 1490 vicuñas entre juveniles y adultas de ambos sexos. La edad de los animales fue definida por la dentición, considerando como adultas a los animales con incisivos definitivos erupcionados ( $>3$ años) ycomojuveniles a los animales sin incisivos definitivos erupcionados ( $<3$ años). Antes de realizar la esquila, se tomaron muestras de fibra $(5 \mathrm{~g})$ de 88 vicuñas ( 36 juveniles y 52 adultas; 57 machos y 31 hembras). La zona corporal del muestro de fibra fue similar a la trabajada por Quispe et al. (2014) para el diámetro medio de fibra (DMF) y finura al hilado $(\mathrm{FH})$. Las muestras fueron colocadas individualmente en bolsas de polipropileno y enviadas al laboratorio de fibras de la Facultad de Zootecnia de la Universidad Nacional Daniel Alcides Carrión (UNDAC), Cerro de Pasco, Perú.
Previo al análisis, se realizó el predescerdado manual, según el procedimiento descrito por Frank et al. (2012). En condiciones estándar de $20 \pm 2{ }^{\circ} \mathrm{C}$ y $65 \pm 5 \%$ de humedad relativa, usando el equipo OFDA 2000 y siguiendo la norma IWTO: 47 (IWTO 47, 2007) se midieron las siguientes características textiles de la fibra predescerdada: DMF, desviación estándar de DMF (DE), coeficiente de variación de DMF (CV), porcentaje de fibras $<30 \mu \mathrm{m}$ (FC), finura al hilado (FH), índice de curvatura (IC), longitud de fibra (LF), así como las variables del perfil de diámetro de fibra (PDF), diámetro máximo del PDF (MAX), diámetro mínimo del PDF (MIN), diferencia entre el diámetro máximo y mínimo del PDF (DIF) y la desviación estándar de PDF (DEP).

\section{Análisis Estadístico}

Para la descripción de las características textiles se determinaron las medidas de tendencia central (promedio) y de dispersión (desviación estándar y coeficiente de variabilidad). El modelo estadístico incluyó como efectos fijos la edad, sexo y la interacción edad:sexo. Para la comparación de medias se utilizó la prueba de Tukey $(\alpha=0.05)$. Las correlaciones fenotípicas entre las características textiles se calcularon mediante el coeficiente de correlación de Pearson $(r)$. Los datos fueron analizados con el software estadístico R (R Core Development Team, 2017).

\section{Resultados y Discusión}

\section{Características Asociadas a la Calidad Textil de la Fibra}

El diámetro medio de fibra $(\mathrm{DMF} \pm \mathrm{DE})$ fue 13.21 \pm 1.41 (Cuadro 1), ligeramente superior al valor de $12.77 \pm 0.52 \mu \mathrm{m}$ y $12.97 \pm$ $0.12 \mu \mathrm{m}$ reportados por Quispe et al. (2018) y Quispe et al. (2011), respectivamente en fibra descerdada. Por otro lado, el DMF fue similar al valor de $13.30 \mu \mathrm{m}$ reportado por 
Olaguivel-Flores et al. (2018) en fibras sin descerdar y al de $13.2 \mu \mathrm{m}$ reportado por Chamut et al. (2016) en fibras secundarias. Debido al descerdado manual, se pueden observar ligeras variaciones, siendo así que Sacchero y Mueller (2005) y Laime et al. (2016) reportan una reducción de $0.3 \mu \mathrm{m}$ y $0.7 \mu \mathrm{m}$, respectivamente, mientras que aumenta el confort de la fibra.

La industria prefiere vellones finos, con un CV menor a $24 \%$. En este estudio se halló mayor uniformidad del DMF y con un CV de $20.44 \%$, similar a $20.02 \%$ y $20.14 \%$ reportados por Trejo et al. (2009) y Quispe et al. (2011), respectivamente. En el caso de alpacas Huacaya, McGregor et al. (2012) reportaron $20.2 \%$, mientras que en llamas, Laime et al. (2016) reportaron $20.34 \%$ en fibra descerdada. Por otro lado, Quispe et al. (2018) reportaron menor CV (4.54\%) en vicuñas de Putina, Puno, mientras OlaguivelFlores et al. (2018) reportaron un valor mayor de CV (30.98\%) en vicuñas de Lucanas, Ayacucho. El sexo y edad no influyó sobre el $\mathrm{CV}$, aunque otros estudios presentan resultados contradictorios (Quispe et al., 2011; Olaguivel-Flores et al., 2018).

La finura al hilado depende de DMF y $\mathrm{CV}$. En este estudio, se obtuvo menor finura al hilado respecto al DMF debido al bajo $\mathrm{CV}$ $(<24 \%)$. La finura al hilado $(12.83 \mu \mathrm{m})$ fue similar a los hallazgos de Quispe et al. (2010, 2011). En forma similar al DMF, la finura al hilado fue más fina en vicuñas juveniles $(-1.42 \mu \mathrm{m})$ respecto a adultas $(\mathrm{p}<0.01)$, similar a los estudios de Quispe et al. (2010, 2011).

El DMF en machos y hembras fue 13.44 $\pm 1.46 \mu \mathrm{m}$ y $12.81 \pm 1.23 \mu \mathrm{m}(\mathrm{p}<0.05)$, respectivamente. La diferencia entre sexos fue similar a la reportada en alpacas (Machaca et al., 2017), probablemente debida a la mayor demanda de nutrientes durante la preñez y lactación. Sin embargo, los trabajos de Quispe (Quispe et al., 2010, 2011, 2018) no hallaron diferencias entre sexos en los valores del DMF. Por otro lado, el DMF en juve- niles y adultas fue $12.26 \pm 1.11 \mu \mathrm{m}$ y $13.88 \pm$ $1.20 \mu \mathrm{m}$, respectivamente $(\mathrm{p}<0.01$; Figura 1$)$, siendo que el DMF aumenta con la edad del animal o el número de esquilas. Además, Gutiérrez et al. (2011) y Pinares et al. (2018) sugieren que algunas fibras secundarias pueden engrosar.

No se observaron diferencias estadísticas por efecto de sexo y edad sobre el índice de curvatura y el porcentaje de fibras $<30 \mu \mathrm{m}$, similar a otros reportes (Quispe et al., 2010, 2011; Olaguivel-Flores et al., 2018), mientras que Machaca et al. (2017) encontraron diferencias estadísticas en fibra de alpacas. En la fibra de vicuña no se observan los rizos; sin embargo, el índice de curvatura fue $73.16 \% \mathrm{~mm}$, variando ente 56 y $97 \% \mathrm{~mm}$ (Figura 2). Otros estudios señalan un índice medio de curvatura $>79^{\circ} / \mathrm{mm}$ (Quispe et al., 2010; Olaguivel-Flores et al., 2018). En el caso de la llama, Laime et al. (2016) reportaron $42.43 \% \mathrm{~mm}$ en fibra descerdada, mientras en alpaca reportaron un valor (bajo) de 37\% $/ \mathrm{mm}$ (Vásquez et al., 2015; Machaca et al., 2017). En estos dos estudios se observa un bajo índice de curvatura que correspondería a fibras con baja cantidad de rizos. En cambio, en lana, mohair y cachemira el rizo fue una característica mejorada genéticamente por selección, donde McGregor et al. (2009) reportaron alta variabilidad fenotípica en cachemira entre 45 y $85^{\circ} / \mathrm{mm}$.

Los hilos más cotizados tienen mayor flexibilidad y menor variación del PDF. La desviación estándar del PDF no mostró variación por efecto de la edad y el sexo. El diámetro mínimo del PDF fue $13.94 \pm 1.30$, mostrando variaciones por edad y sexo, mientras que el diámetro máximo del PDF fue $15.73 \pm 1.98$, mostrando variación por sexo (Cuadro 2; Figura 3). Brown et al. (2000) y Quispe et al. (2008) indican que el PDF puede variar durante el crecimiento de la fibra, dependiendo de factores ambientales como la escasez de alimentos. Por otra parte, la estructura del hilado supone un arreglo longitudinal de fibras para una correcta tor- 
Cuadro 1. Promedio, desviación estándar, coeficiente de variación, valor mínimo y máximo de las características textiles de fibras predescerdadas de 88 vicuñas hembras y machos, juveniles y adultas (Junín, Perú, 2013)

\begin{tabular}{lccccc}
\hline $\begin{array}{l}\text { Características } \\
\text { textiles }^{1}\end{array}$ & Promedio & $\begin{array}{c}\text { Desviación } \\
\text { estándar }\end{array}$ & $\begin{array}{c}\text { Coeficiente de } \\
\text { variación }\end{array}$ & $\begin{array}{c}\text { Valor } \\
\text { mínimo }\end{array}$ & $\begin{array}{c}\text { Valor } \\
\text { máximo }\end{array}$ \\
\hline $\mathrm{DMF}(\mu \mathrm{m})$ & 13.21 & 1.41 & 10.67 & 10.00 & 16.50 \\
$\mathrm{DE}(\mu \mathrm{m})$ & 2.69 & 0.62 & 23.05 & 1.80 & 6.20 \\
$\mathrm{CV}(\%)$ & 20.46 & 4.10 & 20.06 & 15.70 & 41.60 \\
$\mathrm{FC}(\%)$ & 99.96 & 0.30 & 0.30 & 97.30 & 100.00 \\
$\mathrm{FH}(\mu \mathrm{m})$ & 12.83 & 1.47 & 11.42 & 9.50 & 18.75 \\
$\mathrm{IC}(\% \mathrm{~mm})$ & 73.16 & 9.05 & 12.36 & 55.30 & 97.20 \\
$\mathrm{LF}(\mathrm{cm})$ & 2.84 & 0.78 & 27.21 & 1.00 & 4.50 \\
$\mathrm{MAX}(\mu \mathrm{m})$ & 15.50 & 2.45 & 15.78 & 12.10 & 23.80 \\
$\mathrm{MIN}(\mu \mathrm{m})$ & 13.54 & 1.63 & 12.04 & 10.80 & 19.90 \\
$\mathrm{DIF}(\mu \mathrm{m})$ & 1.96 & 1.84 & 94.01 & 0.00 & 11.60 \\
$\mathrm{DEP}(\mu \mathrm{m})$ & 0.84 & 0.68 & 81.04 & 0.10 & 4.80 \\
\hline
\end{tabular}

${ }^{1}$ Diámetro medio de fibra (DMF), desviación estándar de DMF (DE), coeficiente de variación de DMF (CV), porcentaje de fibras $<30 \mu \mathrm{m}(\mathrm{FC})$, finura al hilado (FH), índice de curvatura (IC), longitud de fibra (LF) y diámetro máximo del perfil de fibra (MAX), diámetro mínimo del perfil de fibra (MIN), diferencia entre el diámetro máximo y mínimo del perfil de fibra (DIF), desviación estándar del perfil de diámetro de fibra (DEP)

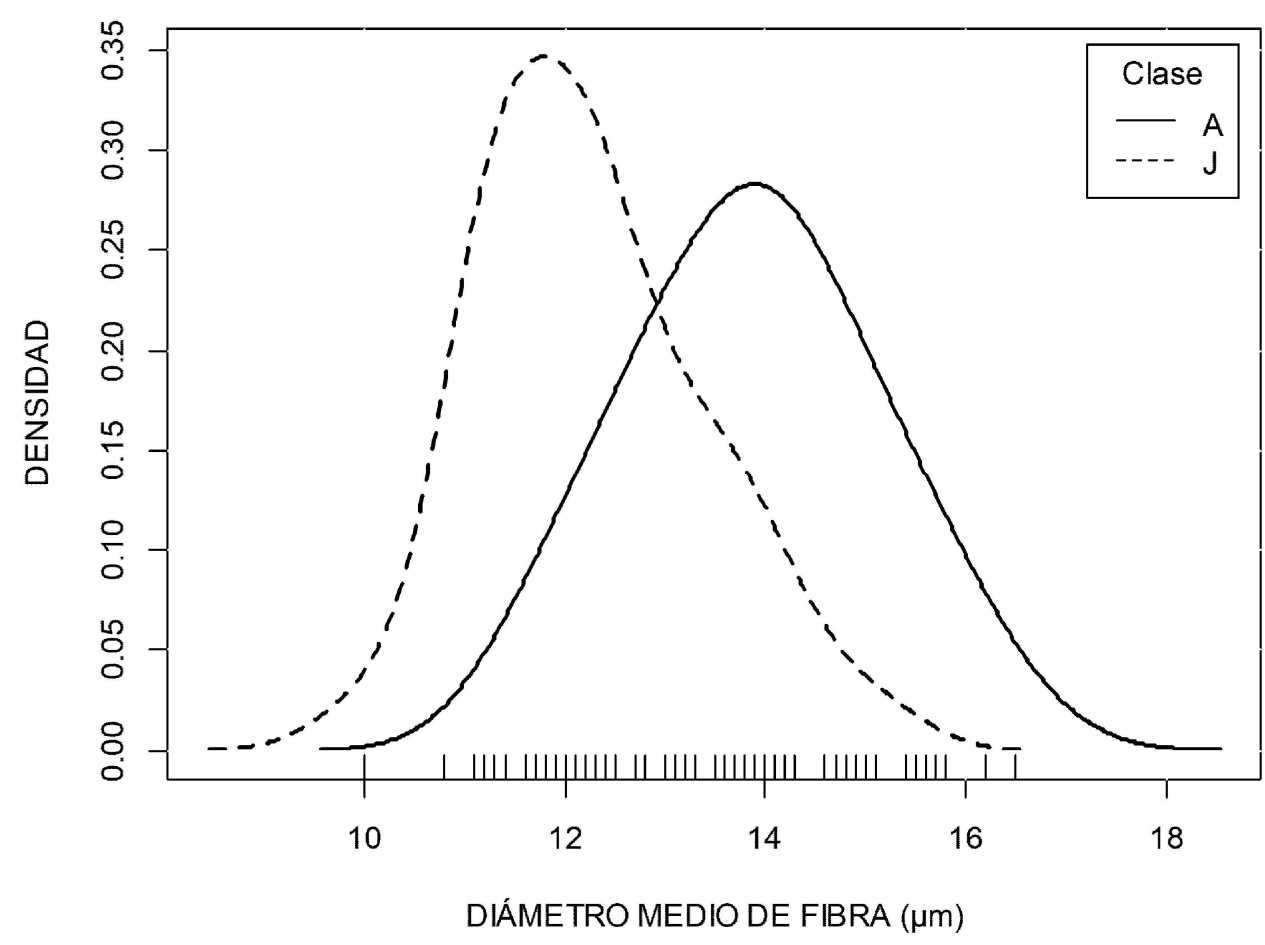

Figura 1. Curva de distribución del diámetro medio de fibra de vicuñas juveniles (J) y adultas (A) de una comunidad campesina de Junín, Perú 


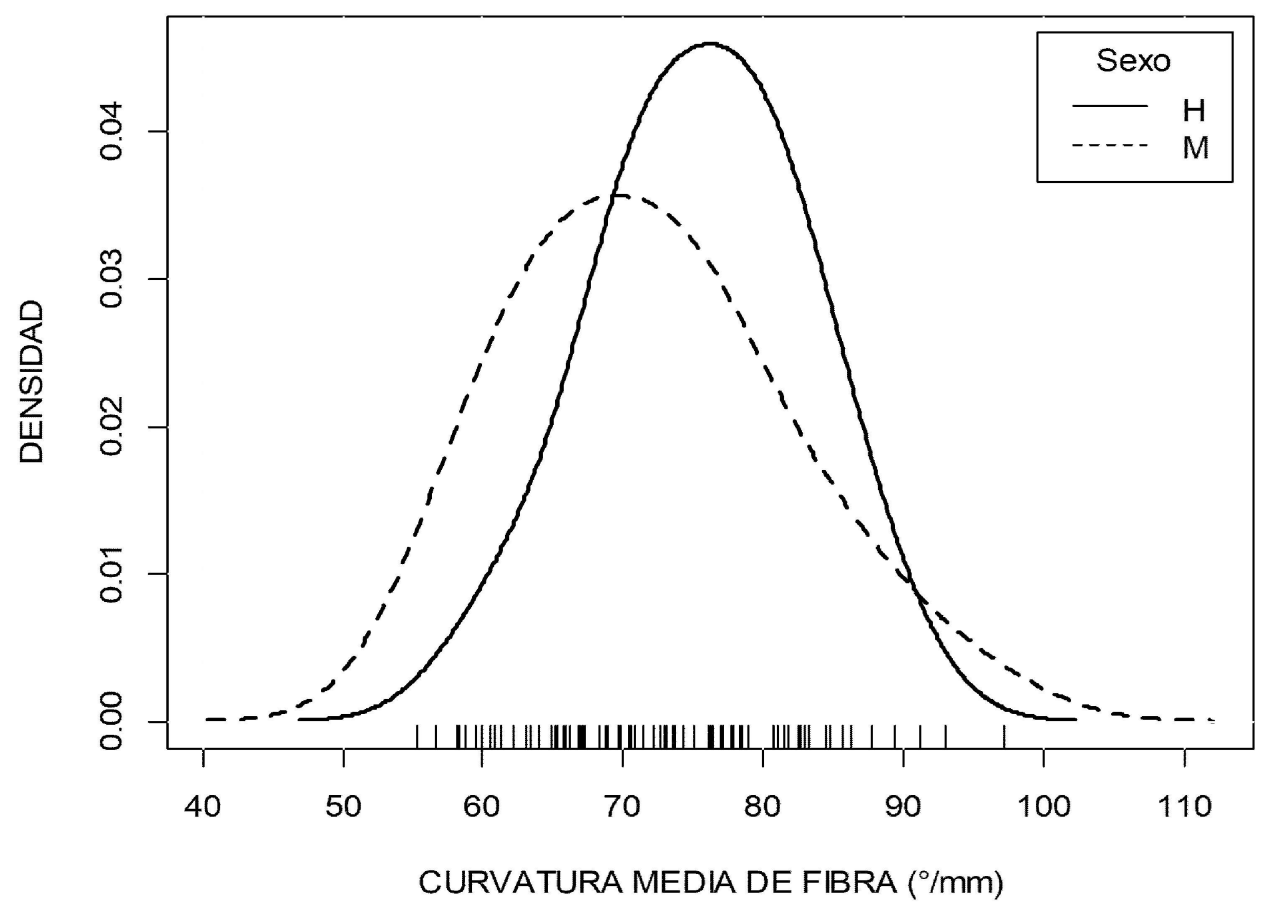

Figura 2. Curva de distribución del índice de curvatura media de fibra de vicuñas hembras $(\mathrm{H})$ y machos $(\mathrm{M})$ de una comunidad campesina de Junín, Perú

Cuadro 2. Promedio \pm desviación estándar del efecto sexo y edad sobre el diámetro medio de fibra (DMF), desviación estándar (DE), coeficiente de variación de DMF (CV), porcentaje de fibras $<30 \mu \mathrm{m}$ (FC), finura al hilado (FH), índice de curvatura (IC), longitud de fibra $(\mathrm{LF})$ y variables ${ }^{1}$ de perfil de diámetro de fibra (PDF) de vicuñas

\begin{tabular}{|c|c|c|c|c|c|c|c|c|c|c|c|c|}
\hline Factor & $\mathrm{n}$ & $\begin{array}{l}\text { DMF } \\
(\mu \mathrm{m})\end{array}$ & $\begin{array}{c}\mathrm{DE} \\
(\mu \mathrm{m})\end{array}$ & $\begin{array}{l}\text { CV } \\
(\%)\end{array}$ & $\begin{array}{l}\mathrm{FC} \\
(\%)\end{array}$ & $\begin{array}{c}\text { FH } \\
(\mu \mathrm{m})\end{array}$ & $\begin{array}{c}\mathrm{IC} \\
(\% / \mathrm{mm})\end{array}$ & $\begin{array}{l}\mathrm{LF} \\
(\mathrm{cm})\end{array}$ & $\begin{array}{c}\text { MAX } \\
(\mu \mathrm{m})\end{array}$ & $\begin{array}{l}\text { MIN } \\
(\mu \mathrm{m})\end{array}$ & $\begin{array}{l}\text { DIF } \\
(\mu \mathrm{m})\end{array}$ & $\begin{array}{l}\text { DEP } \\
(\mu \mathrm{m})\end{array}$ \\
\hline Sexo & & $*$ & ns & ns & Ns & ns & ns & ns & $* *$ & $* *$ & ns & ns \\
\hline \multirow[t]{2}{*}{ Macho } & 57 & 13.44 & 2.70 & 20.18 & 99.98 & 13.02 & 71.88 & 2.75 & 15.98 & 13.98 & 2.01 & 0.88 \\
\hline & & \pm 1.46 & \pm 0.63 & \pm 4.04 & \pm 0.12 & \pm 1.50 & \pm 9.75 & \pm 0.69 & \pm 2.71 & \pm 1.69 & \pm 2.03 & \pm 0.76 \\
\hline \multirow[t]{2}{*}{ Hembra } & 31 & 12.81 & 2.67 & 20.97 & 99.91 & 12.49 & 75.51 & 3.00 & 14.62 & 12.75 & 1.87 & 0.75 \\
\hline & & \pm 1.23 & \pm 0.62 & \pm 4.24 & \pm 0.48 & \pm 1.36 & \pm 7.15 & \pm 0.90 & \pm 1.56 & \pm 1.18 & \pm 1.45 & \pm 0.45 \\
\hline Edad & & $* * *$ & ns & ns & ns & $* * *$ & ns & ns & ns & $* * *$ & ns & ns \\
\hline \multirow[t]{2}{*}{ Juveniles } & 36 & 12.26 & 2.62 & 21.41 & 100 & 11.99 & 74.93 & 2.86 & 15.16 & 12.97 & 2.20 & 0.96 \\
\hline & & \pm 1.11 & \pm 0.45 & \pm 3.40 & \pm 0.00 & \pm 1.10 & \pm 9.93 & \pm 0.60 & \pm 2.99 & \pm 1.89 & \pm 2.31 & \pm 0.92 \\
\hline \multirow[t]{2}{*}{ Adultas } & 52 & 13.88 & 2.74 & 19.80 & 99.93 & 13.41 & 72.93 & 2.81 & 15.73 & 13.94 & 1.79 & 0.75 \\
\hline & & \pm 1.20 & \pm 0.72 & \pm 4.44 & \pm 0.39 & \pm 1.41 & \pm 8.26 & \pm 0.88 & \pm 1.98 & \pm 1.30 & \pm 1.43 & \pm 0.43 \\
\hline $\begin{array}{l}\text { Sexo: } \\
\text { Edad }\end{array}$ & & $\mathrm{ns}$ & ns & ns & ns & $\mathrm{ns}$ & ns & $\mathrm{ns}$ & ns & $\mathrm{ns}$ & ns & $\mathrm{ns}$ \\
\hline
\end{tabular}

${ }^{1}$ Diámetro máximo del PDF (MAX), diámetro mínimo del PDF (MIN), diferencia entre el diámetro máximo y mínimo del PDF (DIF) y desviación estándar del PDF (DEP)

$\mathrm{p}<0.001(* * *), p<0.01(* *), p<0.05(*)$, sin significancia (ns) 


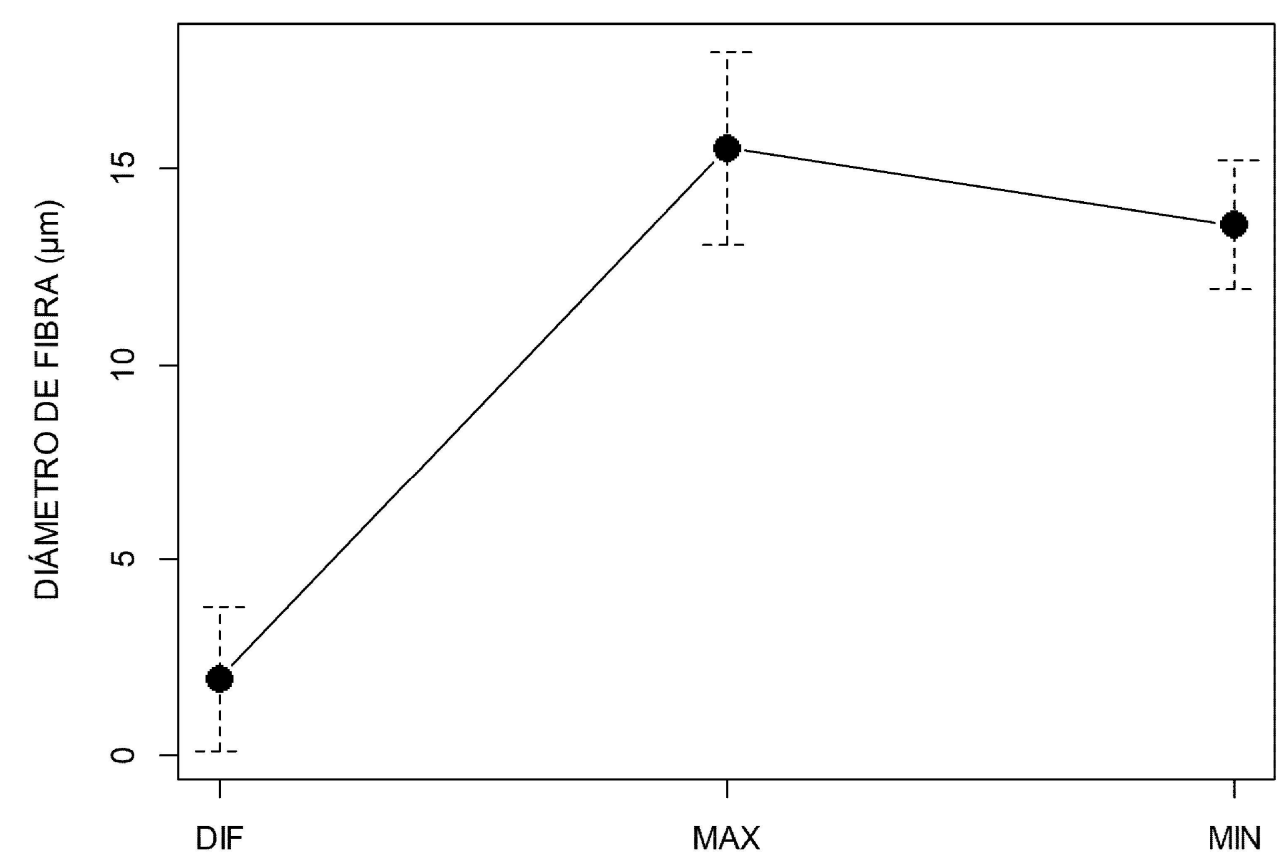

Figura 3. Promedio \pm error estándar del diámetro máximo del perfil de diámetro de fibra $-\mathrm{PDF}$ (MAX), diámetro mínimo del PDF (MIN), diferencia entre el diámetro máximo y mínimo de la fibra (DIF) en vicuñas $(n=88)$ de una comunidad campesina de Junín, Perú

sión del hilo. El rango de variación de longitud media de fibra (LF) fue entre 1.5 y $4.5 \mathrm{~cm}$, sin diferencia por efecto de edad y sexo; sin embargo, Quispe et al. (2018) reportaron variación de LF en crías, juveniles y adultas. En promedio la LF se encuentran dentro del límite de lo aceptable por la industria y depende de la frecuencia en que los animales son esquilados y, probablemente, también depende de otros factores como la nutrición.

\section{Correlaciones Fenotípicas entre las Prin- cipales Características de la Fibra de Vi- cuña}

Se observaron correlaciones fenotípicas positivas y negativas de magnitud intermedia a alta, entre las características textiles de fibra predescerdada de vicuña (Cuadro 3), similar al trabajo de Olaguivel-Flores et al. (2018). La correlación fenotípica entre el DMF y finura al hilado fue 0.92 , similar a la correlación de 0.98 reportada por Quispe et al. (2011) y 0.99 reportada por Vásquez et al. (2015) en fibra de alpacas Huacaya.

Asimismo, se halló una correlación significativa y positiva del DMF con desviación estándar del diámetro (DE), diámetro máximo del PDF (MAX) y mínimo del PDF (MIN). La correlación entre finura al hilado y CV fue positiva (0.27) diferente a -0.30 reportada por Quispe et al. (2011). La diferencia entre MAX y MIN del PDF (DIF) mostró una correlación positiva con MAX y desviación estándar del PDF. También, las correlaciones fenotípicas fueron positivas entre la finura al hilado y DE, CV y DE, DIF y DE, similares al trabajo de Olaguivel-Flores et al. (2018). Por otro lado, las correlaciones fenotípicas fueron negativas y significativas para el porcentaje de fibras $<30 \mu \mathrm{m}$ con CV, DE y finura al hilado. Estos valores permiten sugerir que al disminuir la finura al 
Cuadro 3. Coeficientes de correlación entre el porcentaje de fibras $<30 \mu \mathrm{m}$, diámetro medio de fibra (DMF), desviación estándar (DE), coeficiente de variación de DMF (CV), finura al hilado (FH), índice de curvatura (IC), longitud de fibra (LF) y variables ${ }^{1}$ de perfil de diámetro de fibra (PDF) de vicuñas de una comunidad campesina de Junín, Perú

\begin{tabular}{|c|c|c|c|c|c|c|c|c|c|}
\hline Variable & $\mathrm{CV}$ & $\mathrm{DE}$ & DMF & FH & IC & LF & MAX & MIN & DEP \\
\hline$\%$ fibras & $* * *$ & $* * *$ & ns & $* * *$ & ns & $* *$ & ns & ns & ns \\
\hline \multirow[t]{2}{*}{$<30 \mu \mathrm{m}$} & $<.0001$ & $<.0001$ & 0.1592 & $<.0001$ & 0.2475 & 0.0043 & 0.5331 & 0.6806 & 0.1978 \\
\hline & -0.68 & -0.72 & -0.15 & -0.45 & 0.13 & 0.30 & 0.07 & -0.04 & 0.14 \\
\hline \multirow[t]{3}{*}{$\mathrm{CV}$} & & $* * *$ & ns & $*$ & ns & $* *$ & ns & ns & ns \\
\hline & 1 & $<.0001$ & 0.2094 & 0.0103 & 0.7579 & 0.0063 & 0.6831 & 0.7979 & 0.7745 \\
\hline & & 0.88 & -0.14 & 0.27 & -0.03 & -0.29 & -0.04 & -0.03 & -0.03 \\
\hline \multirow[t]{3}{*}{ DE } & & & $* * *$ & $* * *$ & ns & $* *$ & ns & $* *$ & ns \\
\hline & & 1 & 0.0008 & $<.0001$ & 0.1057 & 0.0067 & 0.1653 & 0.0018 & 0.3630 \\
\hline & & & 0.35 & 0.70 & -0.17 & -0.29 & 0.15 & 0.33 & -0.10 \\
\hline \multirow[t]{3}{*}{ DMF } & & & & $* * *$ & $* *$ & ns & $* * *$ & $* * *$ & ns \\
\hline & & & 1 & $<.0001$ & 0.0024 & 0.9314 & $<.0001$ & $<.0001$ & 0.1963 \\
\hline & & & & 0.92 & $\begin{array}{c}-\mathbf{- 0 . 3 2} \\
* *\end{array}$ & $\begin{array}{c}\mathbf{0 . 0 1} \\
\text { ns }\end{array}$ & $\begin{array}{c}\mathbf{0 . 4 2} \\
* * *\end{array}$ & $\begin{array}{c}\mathbf{0 . 7 7} \\
* * *\end{array}$ & $\begin{array}{c}-\mathbf{0 . 1 4} \\
n s\end{array}$ \\
\hline \multirow{2}{*}{$\mathrm{FH}$} & & & & 1 & 0.0026 & 0.2080 & 0.0004 & $<.0001$ & 0.1427 \\
\hline & & & & & -0.32 & -0.14 & 0.37 & 0.72 & -0.16 \\
\hline \multirow[t]{3}{*}{ IC } & & & & & & ns & $* * *$ & $* * *$ & $*$ \\
\hline & & & & & 1 & 0.1899 & $<.0001$ & $<.0001$ & 0.0202 \\
\hline & & & & & & $\underset{* *}{-\mathbf{0 . 1 4}}$ & $\begin{array}{c}-\mathbf{0 . 4 8} \\
* * *\end{array}$ & $\begin{array}{c}-\mathbf{0 . 4 1} \\
\text { S }\end{array}$ & $\begin{array}{c}\mathbf{- 0 . 2 5} \\
* * *\end{array}$ \\
\hline \multirow{2}{*}{ DIF } & & & & & & 0.0036 & $<.0001$ & 0.9195 & $<.0001$ \\
\hline & & & & & & 0.31 & 0.75 & -0.01 & 0.98 \\
\hline \multirow[t]{3}{*}{ MAX } & & & & & & ns & & $* * *$ & $* * *$ \\
\hline & & & & & & 0.0924 & 1 & $<.0001$ & $<.0001$ \\
\hline & & & & & & 0.18 & & 0.66 & 0.73 \\
\hline
\end{tabular}

${ }^{1}$ Diámetro máximo del PDF (MAX), diámetro mínimo del PDF (MIN), diferencia entre el diámetro máximo y mínimo del PDF (DIF), desviación estándar del PDF (DEP)

El valor de la subfila superior en cada variable: significancia estadística $p<0.001\left({ }^{* * *}\right), p<0.01(* *), p<0.05$ $(*)$, sin significancia (ns)

En la subfila intermedia: probabilidad ( $p$ - valor)

En la subfila inferior: coeficiente de correlación de Pearson

hilado y la variabilidad del DMF se logra aumentar el porcentaje de fibras $<30 \mu \mathrm{m}$; es decir, se incrementa el confort de fibra.

El índice de curvatura mostró una correlación fenotípica negativa de magnitud intermedia (-0.32) con el DMF y con la finura al hilado (-0.32), similar al -0.33 y -0.32 reportados por Quispe et al. (2011), respectivamente, lo cual sugiere un incremento del índice de curvatura cuando el DMF es más fino. Así mismo, Vásquez et al. (2015) y Machaca et al. (2017) reportaron correlaciones de -0.46 y -0.61 en fibra de alpaca Huacaya, respectivamente. Al transformar a $\log _{10}$ el índice de curvatura, McGregor et al. (2012) observaron una relación inversa y proporcional (el índice de curvatura incrementa en $1 \% \mathrm{~mm}$ por la reducción de $1 \mu \mathrm{m}$ del DMF). Además, Frank et al. (2017) reportaron para la fibra de cachemira una correlación de -0.62. 
Respecto a la LF, se obtuvieron correlaciones fenotípicas positivas con \% fibras $<30 \mu$ m y DIF, y negativas con CV y FH. Sin embargo, entre LF y DMF no se observó asociación, aunque Carpio y Santana (1982) reportaron una alta correlación fenotípica entre ambas características (0.87) en vellones de vicuñas machos de Pampa Galeras, indicando una asociación positiva.

\section{Conclusiones}

- El diámetro medio de fibra predescerdada mostró una variación fenotípica por sexo y edad, mas no así el índice medio de curvatura y la longitud media de fibras.

- La fibra predescerdada mostró una mejor uniformidad, buen índice de curvatura $\left(>73.16^{\circ} / \mathrm{mm}\right)$ y confort de fibra (>99.9\%).

- Existen correlaciones fenotípicas favorables, de magnitud intermedia a alta, entre las principales características textiles de fibra predescerdada.

\section{Agradecimientos}

Los autores agradecen a los directivos del Comité de Vicuñas de la Comunidad Campesina Villa de Junín (Junín) por su apoyo en las coordinaciones y por permitir la toma de las muestras de fibra. Asimismo, a la Facultad de Zootecnia de la Universidad Nacional Daniel Alcides Carrión por facilitar el equipo OFDA 2000 para el análisis de las fibras.

\section{Literatura Citada}

1. Brown DJ, Crook BJ, Purvis IW. 2000. Variation in fibre diameter profile characteristics between wool staples in Merino sheep. Wool Tech Sheep Bree 48: 86-93.

2. Carpio M, Santana P. 1982. Estudio preliminar de la longitud y análisis cuticular en la fibra de vicuña. En: In- formes de trabajos de investigación en vicuña. Vol I. Lima, Perú: Univ. Nacional Agraria La Molina. $153 \mathrm{p}$.

3. Chamut S, Cancino AK, BlackDecima P. 2016. The morphological basis of vicuña wool: skin and gland structure in Vicugna vicugna (Molina 1782). Small Ruminant Res 137: 124129. doi: 10.1016/j.smallrumres.2016.03.010

4. Frank EN, Hick MVH, Russano D, Castillo MF, Prieto A, Aisen EG 2017. Sources of variation in fibre production and quality traits source of variation in down-bearing Patagonian goats and mplications for developing a cashmere industry. Small Ruminant Res 150: 6069. doi: 10.1016/j.smallrumres.2017.03.003

5. Frank EN, Hick MVH, Adot OG. 2012. Determination of dehairing, carding, combing and spinning difference from llama type of fleeces. Int J Appl Sci Technol 2: 61-70.

6. Gutiérrez JP, Varona L, Pun A, Morante R, Burgos A, Cervantes I, Pérez-Cabal MA. 2011. Genetic parameters for growth of fiber diameter in alpacas. J Anim Sci 89: 2310-2315. doi: $10.2527 /$ jas.2010-3746

7. IWTO-47. 2007. Measurement of the mean and distribution of fibre diameter of wool using an optical fibre diameter analyser (OFDA). International Wool Textile Organisation.

8. Kasterine A, Lichtenstein G. 2018. El comercio de fibra de vicuña: consecuencias para la conservación y los medios de vida de las poblaciones rurales. Ginebra, Suiza: Centro de Comercio Internacional (ITC). [Internet]. Disponible en: $\mathrm{http}$ //www.intracen.org/uploadedFiles/ intracenorg/Content/Publications/ Vicuna_trade_spanish\%20Low-res.pdf

9. Laime FM, Pinares $R$, Paucara V, Machaca V, Quispe EC. 2016. Características tecnológicas de la fibra de llama (Lama glama) Chaku antes y después de descerdar. Rev Inv Vet Perú 27: 209-217. doi: 10.15381/rivep.v27i2.11643 
10. McGregor B. 2018. Physical, chemical, and tensile properties of cashmere, mohair, alpaca, and other rare animal fibers. In: Hand book of properties of textile and technical fibers. 2nd ed. Elsevier. p 105-136.

11. McGregor BA, Ramos HE, Quispe EC. 2012. Variation of fibre characteristics among sampling sites for Huacaya alpaca fleeces from the High Andes. Small Ruminant Res 102: 191196. doi: 10.1016/j.smallrumres.2011.07.016

12. McGregor BA, Kerven C, Toigonbaev $S .2009$. Sources of variation contributing to production and quality attributes of Kyrgyz cashmere in Osh and Naryn provinces: implication for industry development. Small Ruminant Res 84: 89-99. doi: 10.1016/j.smallrumres.2009.06.008

13. Machaca V, Bustinza AV, Corredor FA, Paucara V, Quispe EC, Machaca R. 2017. Características de la fibra de alpaca Huacaya de Cotaruse, Apurímac, Perú. Rev Inv Vet Perú 28: 843-851. doi: 10.15381/rivep.v28i4.13889

14. Olaguivel-Flores CA, Bujaico $N$, Mamani R, Huamán L. 2018. Características de calidad de la fibra de vicuñas peruanas. En: VIII Congreso Mundial sobre Camélidos. Oruro, Bolivia.

15. Pinares $R$, Gutiérrez $G A, C r u z A$, Morante R, Cervantes I, Burgos A, Gutiérrez JP. 2018. Heritability of individual fiber medullation in Peruvian alpacas. Small Ruminant Res 165: 93-100. doi: 10.1016/j.smallrumres.-2018.04.007

16. Piazza A. 2013. Vicuña: the Queen of the Andes. Skira. 180 p.

17. Quispe JE, Herrera T, Apaza E, Clavetea L, Maquera Z. 2018. Características tecnológicas de la fibra de vicuñas en semicautiverio de la multicomunal Picotani-Región Puno. Rev Inv Vet Perú 29: 522-532. doi: 10.15381/ rivep.v29i2.14475
18. Quispe E, Sánchez F, Filella J, Alfonso $L$. 2014. Variation of commercially important characteristics among sam-pling sites for vicuña (Vicugna vicugna mensalis) fleeces. J Camelid Sci 7: 1-14.

19. Quispe EC, Ramos H, Mayhua P, Alfonso L. 2011. Calidad de la fibra de vicuña (Vicugna vicugna mensalis) bajo condiciones de crianza en semicautiverio en Huancavelica-Perú. En: Quispe EC, Alfonso L (eds). Aportes del PROCASUD al conocimiento de la alpaca y la vicuña. Huancavelica, Perú: Nueva Imagen XXI. p 185-201.

20. Quispe E, Ramos H, Mayhua P, Alfonso L. 2010. Fibre characteristics of vicuña (Vicugna vicugna mensalis). Small Ruminant Res 93: 64-66. doi: 10.1016/j.smallrumres.2010.03.019

21. Quispe E, Rodríguez T, Iñiguez L, Mueller J. 2009. Producción de fibra de alpaca, llama, vicuña y guanaco en Sudamérica. Anim Genetic Resources 45: 1-14. doi: 10.1017/S1014233909990277

22. Quispe EC, Paúcar R, Poma A, Sacchero DM, Mueller JP. 2008. Perfil del diámetro de fibras en alpacas. En: Quispe EC (ed). Biotecnología Aplicada en Camélidos Sudamericanos Huancayo. Edición Gráfica Industrial. p. 131-142.

23. $R$ Core Team. 2017. R: a language and environment for statistical computing. $\mathrm{R}$ Foundation for Statistical Computing, Vienna, Austria. [Internet]. Disponible en: http://www.R-project.org/

24. Sacchero DM, Mueller JP. 2005. Determinación de calidad de vellones de doble cobertura tomando al vellón de vicuña (Vicugna vicugna) como ejemplo. Rev Inv Agro 34: 143-159.

25. Trejo WC, Baquerizo MR, Palacios GP. 2009. Evaluación del diámetro, longitud y rendimiento al lavado de la fibra de vicuña en el patronato del parque de las leyendas. Anales Científicos 70: 4550. doi: 10.21704/ac.v70i1.71 
26. Vásquez RA, Gómez OE, Quispe EC. 2015. Características tecnológicas de la fibra blanca de alpaca Huacaya en la zona altoandina de Apurímac. Rev Inv Vet Perú 26: 213-222. doi: 10.15381/ rivep.v26i2.11020
27. Wheeler JC, Laker J. 2009. The vicuña in the Andean Altiplano. In: Gordon IJ (ed). The vicuña. The theory and practice of community-based wildlife management. Boston, USA: Springer. $p$ 21-34. 